\title{
Equilibrium States of an Ising Ferromagnet in the Low Temperature Region
}

\author{
D. Capocaccia
}

Istituto di Fisica, Università degli Studi di Roma, Italy

M. Cassandro

Istituto di Fisica, Università degli Studi di Roma, Italy and Istituto Nazionale di Fisica Nucleare, Sezione di Roma

\section{G. Ciccotti}

Istituto di Fisica, Università degli Studi di Roma, Italy

Received July 1, 1972

\begin{abstract}
We investigate low temperature properties of an Ising ferromagnet when the nearest neighbour coupling constant is dominant. We show that all the translationally invariant equilibrium states are a superposition of only two extremal states.
\end{abstract}

\section{Introduction}

The existence of a phase transition has been proved for a large class of spin systems on a lattice $[1,2]$, but the determination of the number of pure phases cohexisting below the critical temperature is still an open problem. Only recently Gallavotti and Miracle Solè [3] have shown, for the Ising ferromagnet with only nearest neighbour interaction, that at low enough temperature all translationally invariant equilibrium states can be expressed as a superposition of only two extremal states. The aim of this paper is to extend their result to any finite range pair potential, provided that nearest neighbours interaction is negative, and "dominant" 1 .

We consider an Ising spin system, with a pair interaction, enclosed in a square box $\Lambda$ with side $L$, on a two dimensional square lattice. The probability of a spin configuration in the box, given a spin configuration outside, is proportional to:

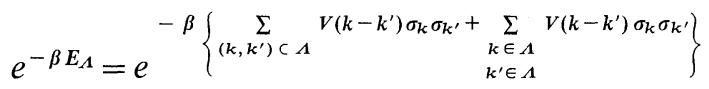

\footnotetext{
${ }^{1}$ The meaning of this term is similar to that used in [1], see formula (2).
} 
where $V(k)$ is such that

$$
\begin{aligned}
& V(k)=V(-k) ; \quad V(k)=-J \text { for }|k|=1 \\
& V(k)=0 \text { if }\left|k_{x}\right|+\left|k_{y}\right|>R \\
& J-\frac{1}{2} \sum_{k:|k|>1}|k||V(k)|=J-D=\alpha>0 .
\end{aligned}
$$

A boundary condition for the box $\Lambda$ is specified by giving a probability distribution $b_{\Lambda}$ for the configurations outside $\Lambda$.

Recalling that an equilibrium state of an infinite system can be defined as a family of translationally invariant correlation functions $\left\{\left\langle\sigma_{A}\right\rangle_{b}\right\}$ obtained as the limit of correlation functions

$$
\left.{\overline{\left\langle\sigma_{A}\right.}}_{\Lambda, b_{A}}=\overline{\left\langle\prod_{k \in A} \sigma_{k}\right\rangle}\right\rangle_{\Lambda, b_{\Lambda}}
$$

(where the bar means average over translations of $A$ in $\Lambda$ ) for a sequence of finite boxes with suitable boundary conditions $([3,4])$, the main result of this paper can be formulated in the following way

Theorem. If $\beta \alpha>2 R \ln 2+\frac{R+1}{2} \ln 3$ any translationally invariant equilibrium state $\left\{\left\langle\sigma_{A}\right\rangle_{b}\right\}$ is given, for some $\alpha_{b}, 0 \leqq \alpha_{b} \leqq 1$ by:

$$
\left\langle\sigma_{A}\right\rangle_{b}=\alpha_{b}\left\langle\sigma_{A}\right\rangle_{+}+\left(1-\alpha_{b}\right)\left\langle\sigma_{A}\right\rangle_{-}
$$

the subscripts $+(-)$ indicate a sequence of boundary conditions defined by putting all spins outside $\Lambda$ equal to $+1(-1)$. A similar theorem has been proved by Gallavotti and Miracle Solè [3] for the case $R=1^{2}$. Following their approach, we will prove two lemmas, by means of which the theorem is easily obtained. The proof of the theorem is unaffected by the presence of longer range interactions, and we refer for it to [3].

In Section 1, we will only sketch the underlying physical idea and formulate the two lemmas.

\section{Section 1}

Consider a fixed configuration, $\tau$, of spin outside $\Lambda$ : it is well known that we can associate to each spin configuration a set of disjoint, self avoiding lines (contours) separating positive from negative spins (for a discussion of possible ambiguites see Ref. [5]).

Some of these lines are closed, the others that we will call $\lambda$ 's, begin and end on the boundary of $\Lambda$. These $\lambda$ lines, whose number is fixed

${ }^{2}$ Their result is stronger because they get $\beta \alpha>\ln 3$. The lack of continuity between the two results is due to the different techniques used. 
once $\tau$ is fixed, divide $\Lambda$ into regions $\vartheta^{+}$'s and $\vartheta^{-}$'s, with spins along the boundaries simultaneusly fixed to be all + and - respectively. Assume now as proven that:

a) In any typical configuration ${ }^{3}$ the $\vartheta^{ \pm}$'s are "large", i.e. the area of the region near the boundaries, where the boundary effects are noticeable, is neglegible;

b) When $A$ is far enough from the boundary of $\vartheta^{ \pm}$

$$
\left\langle\sigma_{A}\right\rangle_{\vartheta^{ \pm}, b_{\vartheta^{ \pm}}} \simeq\left\langle\sigma_{A}\right\rangle_{ \pm} \text {. }
$$

When $\Lambda$ is large enough, the dominat contribution to the average over translations comes, according to a), from points in the middle of large regions $\vartheta^{ \pm}$, assumption b) guarantees then that the average value of the spins products is nearly the value of the corresponding pure phase.

The validity of a) and b) relies on the following two lemmas.

Lemma I. Suppose the spin configurations in $\Lambda$ are given the weight (1) and call $p\left(b_{A}, L^{4 / 3}\right)$ the probability that the total lenght of $\lambda$ lines, for a given boundary condition $b_{A}$, be greater than $L^{4 / 3}$; if $\beta \alpha>\ln 3$

$$
p\left(b_{A}, L^{4 / 3}\right) \leqq \varepsilon(L)
$$

where $\varepsilon(L)$ is a function, not depending on the choice of $b_{A}$ and tending to zero as $L \rightarrow \infty$.

Lemma II. Call $\vartheta^{+}$a region, in the spin lattice, such that all the spins adjacent to its boundary from inside are positive; for all probability distribution $b_{\vartheta^{+}}$on spin configurations outside $\vartheta^{+}$, we have, if: $\beta \alpha>2 R \ln 2$ $+\frac{R+1}{2} \ln 3$

$$
\left|\left\langle\sigma_{A}\right\rangle_{\vartheta^{+}, b_{\vartheta^{+}}}-\left\langle\sigma_{A}\right\rangle_{+}\right| \leqq f(N(A), d, \beta)
$$

where $d$ is the distance of $A \subset \vartheta^{+}$from the boundary of $\vartheta^{+}, N(A)$ is the number of points in $A$, and $f(N(A), d, \beta)$ tends to 0 as $d \rightarrow \infty$.

A similar result holds for $\vartheta^{-}$.

The proof of Lemma I is similar to the one of Ref. [3] and it is given in Appendix 1.

Concerning Lemma II, things are more complicate. In the Ising ferromagnet case, with only n.n. interaction the presence of arbitrary spin configurations beyond the boundary is immaterial, and the second Griffith's inequality can be used to get information on the thermodynamic limit of spin correlation functions. When longer range forces

${ }^{3}$ A set of configurations consists of typical configurations if its probability tends to 1 as $L$ tends to infinity. 
are included Griffith's inequalities can't be used to get a relation between finite systems of different size for the class of boundary conditions of Lemma II, even if the spin interaction is always negative and Fortuin generalization [6] does not seem to be useful.

In the hypothesis of Lemma II, however, only closed contours are present in $\vartheta^{ \pm}$, and techniques introduced by Minlos and Sinai can be used to study the infinite volume limit of contours correlation functions [7] and prove the lemma. In the next section we will express spin correlation functions in terms of a special class of contours correlation functions. Their properties will then be used to prove Lemma II.

\section{Section 2}

Consider a region $\vartheta^{ \pm}$and an arbitrary probability distribution $b_{\vartheta^{ \pm}}$ on the spin configurations outside it. A spin configuration inside $\vartheta^{ \pm}$ corresponds to a set of closed contours.

In any configuration we can consider the subset of outer contours (i.e. the contours that may be connected to the boundary of $\vartheta^{ \pm}$by a lattice path not intersecting any other contour) as the union of disjoint chains $C_{1}, \ldots, C_{n}$; a chain $C$ is defined as the smallest set of outer contours such that if two outer contours may be connected by a lattice walk shorter than $R$, they belong to the same chain.

Two chains are compatible if they can be found in the same configuration as disjoint chains.

According to this definition, two spin inside different chains are not interacting: the interaction between chains is only due to the excluded volume.

Let $C_{1}, \ldots, C_{n}$ be a set of compatible chains, in $\vartheta^{ \pm}$, and $X$ a set of lattice points external to all the contours of the set.

Let $\varrho_{\vartheta^{ \pm}, b_{\vartheta}}\left(C_{1}, \ldots, C_{n} ; X\right)$ be the probability of finding any configuration in $\vartheta^{ \pm}$containing $C_{1}, \ldots, C_{n}$ and such that the points $x \in X$ are all external to any contour of the configuration. As a consequence of the fact that spins inside different outer contours belonging to compatible chains are not interacting, a spin correlation function can be written as

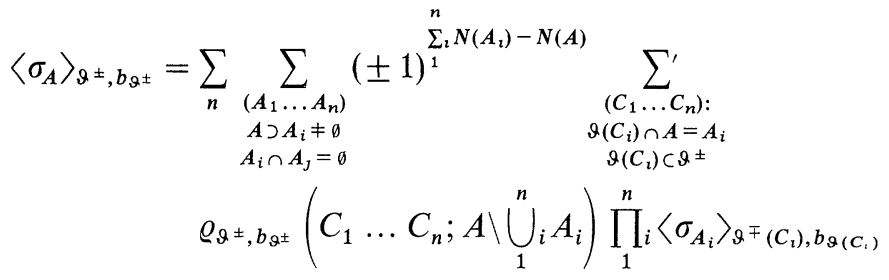


where $\vartheta\left(C_{i}\right)$ is the set of lattice points contained in the outer contours of $C_{i}, N\left(A_{i}\right)$ is the number of points in $A_{i}$ and the primed sum is on the sets of $n$ compatible chains: the empty set is present only if $\bigcup_{1}^{n} A_{i}=\emptyset$.

Remark that $b_{9\left(C_{i}\right)} \neq \pm 1$ only when $C_{i}$ lies closer than $R$ to the border.

Let now $\varrho_{\vartheta^{ \pm}, b_{9^{ \pm}}}\left(C_{1} \ldots C_{n}\right)$ be the probability of finding the set $C_{1} \ldots C_{n}$ of compatible chains in any configuration; it is easily seen that:

$$
\begin{aligned}
& \varrho_{\vartheta^{ \pm}, b_{\vartheta^{ \pm}}}\left(C_{1} \ldots C_{n} ; X\right)=\varrho_{\vartheta^{ \pm}, b_{\vartheta^{ \pm}}}\left(C_{1} \ldots C_{n}\right)+\sum_{k \geqq 1}(-1)^{k} \sum_{\left(\tilde{C}_{1}, \ldots, \tilde{C}_{k}\right):} \\
& \vartheta\left(\tilde{C}_{1}\right) \subset \vartheta^{ \pm} ; \\
& \cdot \varrho_{\vartheta^{ \pm}, b_{\vartheta^{ \pm}}}\left(C_{1} \ldots C_{n}, \tilde{C}_{1} \ldots \tilde{C}_{k}\right)
\end{aligned}
$$

where $\left(\tilde{C}_{1} \ldots \tilde{C}_{k}, C_{1} \ldots C_{n}\right)$ are compatible.

The chains correlation functions $\varrho_{\vartheta^{ \pm}, b_{9^{ \pm}}}\left(C_{1} \ldots C_{n}\right)$ are similar to the ones studied by Minlos and Sinai [7], and satisfy the following properties:

IIa) If $\zeta=e^{-\beta \alpha} 3^{\frac{R+1}{2}}<\left(\frac{1}{2}\right)^{2 R}$ for any set of compatible chains $C_{1} \ldots C_{n}$ a function $\varrho\left(C_{1} \ldots C_{n}\right)$ may be uniquely defined, such that

$$
\left|\varrho\left(C_{1} \ldots C_{n}\right)\right|<e^{-\beta \sum_{1}^{n}\left|C_{2}\right|}
$$

where $\left|C_{i}\right|$ is the total length of outer contours belonging to $C_{i}$;

II b) If $\zeta<\left(\frac{1}{2}\right)^{2 R}$ and $\vartheta\left(C_{1}\right), \ldots, \vartheta\left(C_{n}\right)$ are contained in $\vartheta^{ \pm}$

$$
\left|\varrho_{\vartheta^{ \pm}, b_{9^{ \pm}}}\left(C_{1} \ldots C_{n}\right)-\varrho\left(C_{1} \ldots C_{n}\right)\right|<e^{-\beta \alpha \sum_{1}\left|C_{i}\right|} \quad \zeta^{2 \frac{\delta}{R}}
$$

where $\delta$ is the distance of outer contours belonging to $C_{1}, \ldots, C_{n}$ from the boundary of $\vartheta^{ \pm}$.

Proofs of II a and II b are given in Appendix 2.

It follows from II a that, if $\zeta<\left(\frac{1}{2}\right)^{2 R}$ it is possible to define for any set of compatible chains $C_{1} \ldots C_{n}$ not containing the lattice points $X$ :

$$
\varrho\left(C_{1} \ldots C_{n} ; X\right)=\sum_{k}(-1)^{k} \sum_{\substack{\left(\tilde{C}_{1} \ldots \tilde{C}_{k}\right) \\ \gamma\left(\tilde{C}_{n}\right) \cap X \neq \emptyset}} \varrho\left(C_{1} \ldots C_{n} ; \tilde{C}_{1} \ldots \tilde{C}_{n}\right) .
$$

In fact it can be shown using (8), that the r.h.s. in (10) is dominated by an absolutely convergent series, whose sum has an upper bound given by

$(1+\Phi)^{N(x)} e^{-\beta \alpha \sum_{1}\left|C_{i}\right|}$ where $\Phi=\frac{\zeta^{4}}{1-\zeta^{2}}$, and $\sum_{C: Y(C)=x} e^{-\beta \alpha|C|}<\Phi$ (see Appendix 2). 
Two families of infinite volume spin correlation functions can so be defined, through:

$$
\begin{aligned}
& \left\langle\sigma_{A}\right\rangle_{ \pm}=\sum_{n} \sum_{\substack{\left(A_{i} \ldots A_{n}\right) \\
\sigma \neq A_{i} \subset A \\
A_{i} \cap A_{j}=\emptyset}}( \pm 1)^{\sum_{1} N\left(A_{i}\right)-N(A)} \\
& \sum_{\substack{\left(C_{1} \ldots C_{n}\right) \\
\vartheta\left(C_{i}\right) \cap A=A_{l}}} \varrho\left(C_{1} \ldots C_{n} ; A \backslash \bigcup_{1}^{n} A_{i}\right) \Pi_{i}\left\langle\sigma_{A_{i}}\right\rangle_{\vartheta^{\mp}\left(C_{i}\right), \pm}
\end{aligned}
$$

the r.h.s. is in fact dominated by:

$$
(1+\Phi)^{N(A)} \sum_{0}^{N(A)} n\left(\begin{array}{c}
N(A) \\
h
\end{array}\right) \Phi^{n}=(1+\Phi)^{2 N(A)}
$$

as it is easily checked, using the previous definitions, and noting that any set of $n$ chains appearing in (11) can be choosen in at most $\left(\begin{array}{c}N(A) \\ n\end{array}\right)$ ways.

If $\zeta<\left(\frac{1}{2}\right)^{2 R}$ and $\left(\vartheta\left(C_{1}\right), \ldots, \vartheta\left(C_{n}\right)\right)<\vartheta$ an upper bound for the difference between $\varrho_{\vartheta^{ \pm}, b_{\vartheta^{ \pm}}}\left(C_{1} \ldots C_{n} ; X\right)$ and $\varrho\left(C_{1} \ldots C_{n} ; X\right)$ as given by (7) and (10) respectively may be evaluated with standard computations using II b; it results:

$$
\left|\varrho_{\vartheta^{ \pm}, b_{9^{ \pm}}}\left(C_{1} \ldots C_{n} ; X\right)-\varrho\left(C_{1} \ldots C_{n} ; X\right)\right| \leqq e^{-\beta \sum_{1}^{n}\left|C_{2}\right|} F(N(X)) \zeta^{\frac{4}{R}}
$$

where $\Delta$ is the minimum of the distances of $X$ and $C_{1} \ldots C_{n}$ from the boundary of $\vartheta^{ \pm}$, and $F(N(X))$ is finite for finite $X$.

From (6), (11) and (13) it follows finally

$$
\left|\left\langle\sigma_{A}\right\rangle_{\vartheta^{ \pm}, b_{\vartheta^{ \pm}}}-\left\langle\sigma_{A}\right\rangle_{ \pm}\right| \leqq G(N(A)) \zeta^{\frac{d}{R}}
$$

where $G$ is finite for finite $A$, and $d$ is the distance of $A$ from the boundary of $\vartheta$; (14) proves Lemma II.

Remark. Our condition on the pair interaction is probably too strong and with a different definition of the contour could possibly be weakened.

We remark however that the weaker condition assumed by Dobrushin [10] to study the properties of a gas of "extended contours" does not ensure the existence of only two phases in the low temperature region.

Consider a 2-dimensional spin system with only next nearest neighbour (i.e. consisting of two non-interacting sublattices each with only nearest neighbour interaction). If the coupling constant is negative this system satisfies Dobrushin condition but at low temperature at least three pure phases are present. 
The first two have respectively positive and negative magnetization, corresponding to simultaneous positive or negative magnetization in the two sublattices and the third one, with zero magnetization, is a translationally invariant linear combination of the two states corresponding to opposite magnetization in the two sublattices.

Acknowledgement. We are greatly indebted to G. Gallavotti for having proposed the problem and for helpful suggestions. Useful discussions with F. Di Liberto are also acknowledged.

\section{Appendix I}

Consider a fixed configuration $\tau$ of spins outside $\Lambda$ and a spin configuration $\sigma$ inside it. Let $\lambda_{1} \ldots \lambda_{k}$ be the corresponding set of open contours. $\lambda_{1} \ldots \lambda_{k}$ divide $\Lambda$ into regions $\vartheta_{1} \ldots \vartheta_{s}, s \leqq k+1, \bigcup_{i}^{s} \vartheta_{i}=\Lambda$; an index + or - may be assigned to each $\vartheta$ according to the sign of spins $\tau$ adjacent to its boundary, that is the sign of the spins inside $\vartheta$, adjacent to $\lambda$ lines.

Let now $\sigma^{*}$ be the configuration obtained from $\sigma$ by flipping all the spins contained in the regions $\vartheta_{i}^{-}$'s.

According to an argument of Ginibre, Grossmann, and Ruelle [1] one has:

$$
\begin{gathered}
E_{\Lambda}(\sigma \cup \tau)-E_{\Lambda}\left(\sigma^{*} \cup \tau\right) \geqq 2 J\left(\sum_{1}^{k}\left|\lambda_{i}\right|-4 L\right) \\
-2 D\left(\sum_{i}^{k}\left|\lambda_{i}\right|+4 L\right) .
\end{gathered}
$$

The first term on the right hand side is in fact less than the n.n. contribution to energy variation, and the second one has an absolute value larger than the longer range contributions.

(A.1) can be used to obtain an upper bound for the probability of finding $\lambda_{1}, \ldots, \lambda_{k}$ as open contours, provided spins outside $\Lambda$ are fixed.

Observing that if $\lambda_{1} \ldots \lambda_{k}$ are fixed, the spin transformation previously defined can be inverted one has:

$$
p_{\tau}\left(\lambda_{1} \ldots \lambda_{k}\right) \leqq \frac{\sum_{\sigma}^{\prime} e^{-\beta E_{\Lambda}(\sigma \cup \tau)}}{\sum_{\sigma}^{\prime} e^{-\beta E_{\Lambda}\left(\sigma^{*} \cup \tau\right)}} \leqq e^{8 \beta(J+D) L-2 \beta \alpha \sum_{1}^{k}\left|\lambda_{i}\right|}
$$

where the prime means that the sum runs over spin configurations having $\lambda_{1} \ldots \lambda_{k}$ as open contours. 
A set $\lambda_{1} \ldots \lambda_{k}$, with lengths $\ell_{1}, \ldots, \ell_{k}$ may be choosen in at most

$$
\begin{aligned}
& \left(\begin{array}{c}
2 k \\
k
\end{array}\right) 3 \sum_{1}^{n} \ell_{i} \text { ways. Then, if } 3 e^{-2 \beta \alpha}<1 \\
& p\left(\tau, \sum_{1}^{k}\left|\lambda_{i}\right| \geqq L^{4 / 3}\right) \leqq e^{8 \beta(J+D) L}\left(\begin{array}{c}
2 k \\
k
\end{array}\right) \sum_{\substack{\ell_{1} \ldots \ell_{k}: \\
\ell_{i}>0, \sum_{i} \ell_{i} \geqq L^{4 / 3}}}\left(3 e^{-2 \beta \alpha}\right)^{\sum^{\sum_{i} \ell_{i}}} \\
& \leqq e^{8 \beta(J+D) L}\left(\begin{array}{c}
2 k \\
k
\end{array}\right)\left(\begin{array}{c}
L^{4 / 3} \\
k
\end{array}\right)\left(3 e^{-2 \alpha \beta}\right)^{L^{4 / 3}} \sum_{\ell_{1}^{\prime} \ldots \ell_{k}^{\prime}}\left(3 e^{-2 \beta \alpha}\right)^{\sum_{i} \ell_{i}^{\prime}} \\
& \leqq e^{8 \beta(J+D) L}\left(\frac{4}{1-3 e^{-2 \alpha \beta}}\right)^{2 L} \max _{k \leqq 2 L}\left(\begin{array}{c}
L^{4 / 3} \\
k
\end{array}\right)\left(3 e^{-2 \beta \alpha}\right)^{L^{4 / 3}}=\varepsilon(L)
\end{aligned}
$$

where $\varepsilon(L)$ does not depend on $\tau$ and tends to zero as $L \rightarrow \infty$. Note that in getting (A.1) n.n. dominance has been used, while the finite range hypothesis is unessential.

\section{Appendix II}

Consider a region $\vartheta^{+}$, and a fixed configuration $\tau$ of spins outside it. If spin configurations in $\vartheta^{+}$are given the weight: $p_{\tau}(\boldsymbol{\sigma})=\frac{e^{-\beta E_{\vartheta(\sigma)}}}{Z_{\tau}(\vartheta)}$ where: $E_{\vartheta}(\boldsymbol{\sigma})=\sum_{(x, y) \subset \vartheta} V(x-y) \sigma_{x} \sigma_{y}+\sum_{\substack{x \in \vartheta \\ y \notin \vartheta}} V(x-y) \sigma_{x} \tau_{y}$ and

$$
Z_{\tau}(\sigma)=\sum_{\sigma} e^{-\beta E_{\vartheta}(\sigma)}
$$

chains correlation functions may be written as:

$$
\varrho_{\vartheta, \tau}\left(C_{1} \ldots C_{n}\right)=\frac{Z_{\tau}\left(C_{1} \ldots C_{n}\right)}{Z_{\tau}(\vartheta)}
$$

where $Z_{\tau}\left(C_{1} \ldots C_{n}\right)$ is the contribution to $Z_{\tau}(\vartheta)$ due to the configurations containing $C_{1} \ldots C_{n}$.

For each configuration $\boldsymbol{\sigma}$ in the numerator, consider in the denominator the configuration $\boldsymbol{\sigma}^{*}$, obtained by flipping all the spins in $\vartheta\left(C_{1}\right), \ldots, \vartheta\left(C_{n}\right)$ : the correspondence is one to one.

According to the argument of G.G.R., already used in Appendix I: $E_{\vartheta}(\boldsymbol{\sigma})-E_{\vartheta}\left(\boldsymbol{\sigma}^{*}\right) \geqq 2 \alpha \sum_{i}^{n}\left|C_{i}\right|$ and therefore:

$$
\varrho_{\vartheta, \tau}\left(C_{1} \ldots C_{n}\right) \leqq e^{-2 \alpha \sum_{i}^{n}\left|C_{\imath}\right|}
$$


To prove the existence of the limits of the correlations functions and estimate the rate of convergence the procedure is almost standard.

Introduced by Ruelle [8] for a gas system in the continous case and extended by Minlos and Sinai [7] to the "gas of contours" it can be applied to our case with some obvious changes.

Let $\mathscr{B}$ be the Banach space of the functions $\varphi$ defined over the sets of compatible chains, with the norm:

$$
\|\varphi\|=\sup _{n, C_{1} \ldots C_{n}}\left|\varphi\left(C_{1} \ldots C_{n}\right) e^{\beta \alpha \Sigma_{i}\left|C_{i}\right|}\right| .
$$

Define $\lambda \in \mathscr{B}$ as:

$$
\begin{gathered}
\lambda(C)=\frac{z_{+}\left(\vartheta^{-}(C)\right)}{z_{+}\left(\vartheta^{+}(C)\right)} \\
\lambda\left(C_{1}, \ldots, C_{n}\right)=0 \text { if } n>1
\end{gathered}
$$

where the plus subscript to $z$ means that all spins outside $\vartheta^{ \pm}(C)$ are positive. The same argument used for (A.6) yields:

$$
\lambda(C) \leqq e^{-2 \beta \alpha|C|} .
$$

For finite $\vartheta$ and a given $\tau$, also define $\lambda_{\vartheta, \tau} \in \mathscr{B}$ as: $\lambda_{\vartheta, \tau}\left(C_{1} \ldots C_{n}\right)=0$ if $n>1$, or if $C_{i} \nsubseteq \vartheta$ for some:

$$
\lambda_{\vartheta, \tau}(C)=\frac{z_{\tau_{c}}\left(\vartheta^{-}(C)\right)}{z_{\tau_{c}}\left(\vartheta^{+}(C)\right)}
$$

where $\tau_{C}$ is spin configuration outside $C$.

Remark that, if $\vartheta(C) \subset \vartheta$ and $C$ lies farther than $R$ from boundary, $\lambda_{\vartheta, \tau}=\lambda$, moreover: $\lambda_{\vartheta, \tau}(C)<e^{-2 \alpha|C|}$. Let the operator $\chi_{\vartheta}$ be defined as

where

$$
\left(\chi_{\vartheta} \varphi\right)\left(C_{1} \ldots C_{n}\right)=\chi_{\vartheta}\left(C_{1} \ldots C_{n}\right) \varphi\left(C_{1} \ldots C_{n}\right)
$$

$$
\chi_{\vartheta}\left(C_{1} \ldots C_{n}\right)=\prod_{1}^{n} \chi_{\vartheta}\left(C_{i}\right) \text { and } \chi_{\vartheta}(C)= \begin{cases}1 & \text { if } \vartheta(C) \subset \vartheta \\ 0 & \text { otherwise }\end{cases}
$$

Define next the two following operators $A_{\vartheta}$ and $A$ on $\mathscr{B}$

$$
\begin{aligned}
& \left(A_{\vartheta} \varphi\right)\left(C_{1}\right)=\chi_{\vartheta}\left(C_{1}\right) \lambda_{\vartheta, \tau}\left(C_{1}\right) \\
& \cdot\left\{\sum_{k \geqq 1}(-1)^{k} \sum_{\substack{\left(\tilde{C}_{1} \ldots \tilde{C}_{n}\right): \\
\tilde{C}_{\imath} \cap C_{i} \neq \emptyset}} \chi_{9}\left(\tilde{C}_{1} \ldots \tilde{C}_{n}\right) \varphi\left(\tilde{C}_{1} \ldots \tilde{C}_{n}\right)-\sum_{\tilde{C} \subset C_{1}} \chi_{\vartheta}(\tilde{C}) \varphi(\tilde{C})\right\}
\end{aligned}
$$


For $n>1$

$$
\begin{aligned}
& \left(A_{\vartheta} \varphi\right)\left(C_{1} \ldots C_{n}\right)=\chi_{\vartheta}\left(C_{1} \ldots C_{n}\right) \lambda_{\vartheta, \tau}\left(C_{1}\right) \\
& \cdot\left\{\varphi\left(C_{2} \ldots C_{n}\right)+\sum_{k \geqq 1}(-1)^{k} \sum_{\substack{\left(\tilde{C}_{1} \ldots \tilde{C}_{n}\right) \\
\tilde{C}_{i} \cap C_{1} \neq \emptyset}} \chi_{\vartheta}\left(\tilde{C}_{1} \ldots \tilde{C}_{k}\right) \varphi\left(C_{2}, \ldots, C_{n}, \tilde{C}_{2} \ldots \tilde{C}_{k}\right)\right. \\
& \left.-\sum_{\tilde{C} \subset C_{1}} \chi_{\vartheta}(\tilde{C}) \varphi\left(C_{2} \ldots C_{n}, \tilde{C}\right)\right\} \\
& (A \varphi)\left(C_{1}\right)=\lambda\left(C_{1}\right)\left\{\sum_{k \geqq 1}(-1)^{k} \sum_{\substack{\left(\tilde{C}_{1} \ldots \tilde{C}_{k}\right) \\
\tilde{C}_{i} \cap C_{1} \neq \emptyset}} \varphi\left(\tilde{C}_{1} \ldots \tilde{C}_{k}\right)-\sum_{\tilde{C} \subset C_{1}} \varphi(\tilde{C})\right\}
\end{aligned}
$$

and for $n>1$

$$
\begin{aligned}
(A \varphi)\left(C_{1} \ldots C_{n}\right)= & \lambda\left(C_{1}\right)\left\{\varphi\left(C_{2} \ldots C_{n}\right)+\sum_{k \geqq 1}(-1)^{k}\right. \\
& \left.\cdot \sum_{\substack{\left(\tilde{C}_{1} \ldots \tilde{C}_{k}\right) \\
\tilde{C}_{\imath} \cap C_{1} \neq \emptyset}} \varphi\left(C_{2} \ldots C_{n}, \tilde{C}_{1} \ldots \tilde{C}_{k}\right)-\sum_{\tilde{C} \subset C_{1}} \varphi\left(C_{2} \ldots C_{n}, \tilde{C}\right)\right\} .
\end{aligned}
$$

Where the first sum is over the set of contours "intersecting" $C_{1}$ and the second over the contours embracing $C_{1}$.

We say that $C$ "intersects" $C^{\prime}\left(C \cap C^{\prime} \neq \emptyset\right)$ if some outer contour belonging to $C$ intersects an outer contour of $C^{\prime}$, or lies closer than $R$ to it; and that $C$ embrases $C^{\prime}\left(C \subset C^{\prime}\right)$ if all the contours of $C^{\prime}$ are contained in an outer contour of $C$ and lie at a distance larger than $R$ from it.

In our case the number of chains of total length $\ell$ starting from a given point is less than $3^{\frac{R+1}{2} \ell}$, [9]. Then observing that $k$ initial points for a set $\tilde{C}_{1} \ldots \tilde{C}_{k}$ in the first sum may be choosen in at most $\left(\begin{array}{c}2 R\left|C_{1}\right| \\ k\end{array}\right)$ ways and noting that all $\tilde{C}$ of the second sum should contain a given point of $\vartheta\left(C_{1}\right)$, and therefore intersect a straight line starting from it, one finds; a

$$
\begin{aligned}
& \frac{\left|(A \varphi)\left(C_{1} \ldots C_{n}\right)\right|}{\|\varphi\| e^{-2 \alpha \Sigma_{l}\left|C_{i}\right|} \leqq} e^{-\beta \alpha\left|C_{1}\right|} \\
& \cdot\left\{\sum_{0}^{2 R\left|C_{1}\right|}\left(\begin{array}{c}
2 R\left|C_{1}\right| \\
k
\end{array}\right)\left(\sum_{2}^{\infty} \zeta^{2 \ell}\right)^{k}+\sum_{1}^{\infty} \sum_{\ell \geqq x+1} \zeta^{2 \ell}\right\} \\
& \leqq e^{-\beta \alpha\left|C_{1}\right|}\left\{\left(1+\frac{\zeta^{4}}{1-\zeta^{2}}\right)^{2 R\left|C_{1}\right|}+\frac{\zeta^{4}}{\left(1-\zeta^{2}\right)^{2}}\right\}
\end{aligned}
$$


if $\zeta=3^{\frac{R+1}{2}} e^{-\beta \alpha}<1$. An identical inequality holds for $A_{\vartheta}$. Then, if $\zeta<\left(\frac{1}{2}\right)^{2 R}$

$$
\begin{aligned}
\|A\| & =\sup _{\varphi} \frac{\|A \varphi\|}{\|\varphi\|}<1 . \\
\left\|A_{\vartheta}\right\| & =\sup _{\varphi} \frac{\left\|A_{\vartheta} \varphi\right\|}{\|\varphi\|}<1 .
\end{aligned}
$$

The proof of (8) and (9) in Section 2 now proceds exactly on the same lines of Ref. [7] and the reader is referred to the summentioned paper for more details (see Lemma 4, p. $250 \mathrm{ff}$. of Ref. [7]). The simple changes needed to adapt the proof to our case are briefly discussed below: consider the two equations:

$$
\begin{aligned}
& \varphi=\chi_{\vartheta} \lambda_{\vartheta, \tau}+A_{\vartheta} \varphi, \\
& \varphi=\lambda+A \varphi .
\end{aligned}
$$

If $\zeta<\left(\frac{1}{2}\right)^{2 R}$ due to (A.10), solution of (A.11) and (A.11) is unique in $\mathscr{B}$ and it can be shown that (A.11) is identically satisfied by ${ }^{4} \varrho_{\vartheta, \tau}$ and $\varrho_{\vartheta, \tau} \in \mathscr{B}$ due to (A.6). Call $\varrho$ the solution of (A.11)'. If $\zeta<\left(\frac{1}{2}\right)^{2 R}, \eta_{\vartheta, \tau}=\varrho_{\vartheta, \tau}-\chi_{\vartheta} \varrho$ is the unique solution in $\mathscr{B}$ of

where

$$
\eta_{\vartheta, \tau}=\xi_{\vartheta, \tau}+A_{\vartheta} \eta_{\vartheta, \tau}
$$

$$
\xi_{\vartheta, \tau}=\lambda_{\vartheta, \tau}-\chi_{\vartheta} \lambda+\chi_{\vartheta}\left(A_{\vartheta} \chi_{\vartheta}-A\right) \varrho \text {. }
$$

Let now $C_{1} \ldots C_{n}$ be a set of compatible chains, with $\vartheta\left(C_{1}\right) \ldots \vartheta\left(C_{n}\right) \subset \vartheta$, and $\delta$ the distance of $C_{1} \ldots C_{n}$ from the boundary of $\vartheta$. In any term contributing to $\xi_{\vartheta, \tau}\left(C_{1} \ldots C_{n}\right)$ there is at least one chain whose total length is $\ell \geqq \frac{4 \delta}{R}$ (the shorter chain consists of $\frac{\delta}{R}$ outer contours, of minimal length 4). The argument just used to get (A.9) yields:

and

$$
\left|\xi_{\vartheta, \tau}\left(C_{1} \ldots C_{n}\right)\right| \leqq B_{1} e^{-\beta \alpha\left(\left|C_{1}\right|+\cdots+\left|C_{n}\right|\right)} \zeta^{\frac{2 \delta}{R}}
$$

$$
\left|\left(A_{\vartheta} \xi_{\vartheta, \tau}\right)\left(C_{1} \ldots C_{n}\right)\right| \leqq u B_{1} e^{-\beta \alpha \sum_{1}\left|C_{1}\right|} \zeta^{\frac{2 \delta}{R}}
$$

where $u<1$.

${ }^{4}$ All the elements of the set of configurations contributing to $Z_{\tau}\left(C_{2} \ldots C_{n}\right)$ and not contributing to $Z_{\tau}\left(C_{1} \ldots C_{n}\right)$ should contain at least i) an outer contour $\gamma$ such that $\vartheta(\gamma) \subset \vartheta\left(C_{1}\right)$; ii) an outer contour intersecting some outer contour of $C_{1}$ or lying closer than $R$ to it; iii) an outer contour embracing all outer countors of $C_{1}$; i) and iii), and ii) and iii) can't happen simultaneously. 
The inequality

$$
\left|\varrho_{\vartheta^{ \pm}, \tau}\left(C_{1} \ldots C_{n}\right)-\left(\chi_{\vartheta} \varrho\right)\left(C_{1} \ldots C_{n}\right)\right|<B_{2} e^{-\beta \alpha \sum_{1}^{n}\left|C_{z}\right|} \zeta^{\frac{2 \delta}{R}}
$$

is then satisfied if $\left\|A_{\vartheta}\right\|<1$, that is if $\zeta<\left(\frac{1}{2}\right)^{2 R}$.

Starting with a region $\vartheta^{-}$everything proceeds in the same way; one gets again Eq. (A.11)', because $\frac{Z_{-}\left(\vartheta^{+}(C)\right)}{Z_{-}\left(\vartheta^{-}(C)\right)}=\frac{Z_{+}\left(\vartheta^{-}(C)\right)}{Z_{+}\left(\vartheta^{+}(C)\right)}$. The IIb is so proven.

\section{References}

1. Ginibre, J., Grossmann, A., Ruelle, D.: Commun. math. Phys., 3, 187 (1967).

2. Dobrushin, R. L.: Teor. Verojatnost. Primenen. 9, 626 (1964). — Griffiths, R.: Phys. Rev. 136, A 437 (1964).

3. Gallavotti, G.: Miracle Solé S. Phys. Rev. 5, B 2555 (1972).

4. Lanford, D., Ruelle, D.: Commun. math. Phys. 13, 194 (1969).

5. Gallavotti, G., Martin-Lof, A.: Commun. math. Phys. 25, 87 (1972).

6. Fortuin, C.M.: Kasteleyn,P.W., Ginibre,J.: Commun. math. Phys. 22, 89 (1971).

7. Minlos, R.A., Sinai, Ja G.: Trans. Moscow Math. Soc. 17, 237 (1967).

8. Ruelle,D.: Statistical Mechanics Chapter IV, New York: W. A. Benjamin 1969.

9. Ruelle, D.: Phys. Rev. Letters 27, 1040 (1971).

10. Dobrushin, R.L.: Proc. Fifth Berkeley Sympos. on Mathematical Statistics and Probability, 1965, Berkeley: Univ. of California Press., 1967.

D. Capocaccia

Istituto di Fisica

Università degli Studi

di Roma

I-00185 Roma, Italia

Piazzale delle Scienze 5 